\title{
Evolución del perfil profesional del community manager durante la década 2009-2018
}

\section{Community manager's professional profile evolution along the decade 2009-2018}

\author{
Luis Mañas-Viniegra; Isidro Jiménez-Gómez
}

Cómo citar este artículo:

Mañas-Viniegra, Luis; Jiménez-Gómez, Isidro (2019). “Evolución del perfil profesional del community manager durante la década 2009-2018". El profesional de la información, v. 28, n. 4, e280403.

https://doi.org/10.3145/epi.2019.jul.03

Artículo recibido el 11-12-2018

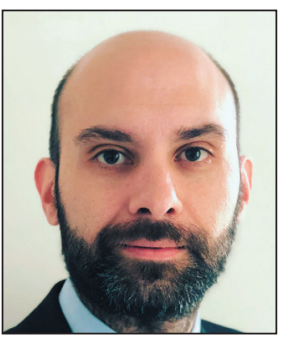

Luis Mañas-Viniegra $\square$ http://orcid.org/0000-0001-9129-5673

Universidad Complutense de Madrid Facultad de Ciencias de la Información Departamento de Ciencias de la Comunicación Aplicada Avda. Complutense, 3. 28040 Madrid, España Imanas@ucm.es

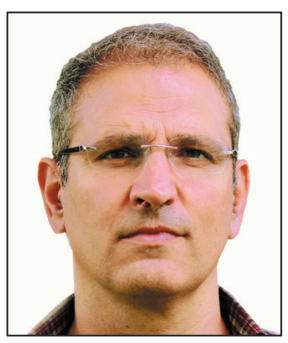

Isidro Jiménez-Gómez http://orcid.org/0000-0001-7372-7276

Universidad Complutense de Madrid Facultad de Ciencias de la Información Departamento de Ciencias de la Comunicación Aplicada Avda. Complutense, 3. 28040 Madrid, España isidroji@ucm.es

\section{Resumen}

La especialización de community manager apenas está presente en los planes de estudio de los grados en Comunicación, a pesar de la creciente importancia de las redes sociales como fuente de empleo y de que el $75 \%$ de las ofertas que incorporan tareas de community manager correspondan a un perfil exclusivo y diferenciado de otros digitales. A partir de una muestra formada por las 731 ofertas publicadas con funciones de community manager en domestika.org, el principal portal especializado en el sector, se realiza un análisis de este perfil profesional durante la década 2009-2018. La gestión de redes sociales ha dejado de corresponder a programadores y diseñadores web para convertirse en un perfil profesional de Comunicación y cada año se exigen más competencias y habilidades al community manager, aunque en un contexto de mayor precariedad laboral. Además, parece estar evolucionando hacia la gestión de influencers.

\section{Palabras clave}

Perfiles profesionales; Community managers; Gestión de comunidades; Redes sociales; Medios sociales; Comunicación digital; Competencias profesionales; Ofertas de trabajo.

\begin{abstract}
The academic major of community manager is scarcely present in the curricula of Communication degrees, despite the growing importance of social media as a source of employment and the fact that $75 \%$ of the job offers that include community manager tasks have a profile that is exclusive and different from those of other jobs in the digital area. Based on a sample of 731 employment offers with community manager tasks published in domestika.org, the main job portal specializing in this sector, an analysis of this professional profile has been made during the decade 2009-2018. The management of social media is no longer the sole domain of programmers and web designers. In fact, this position has experienced a transformation into a professional Communication profile, and each year more competencies and skills are demanded of the community manager, yet within a context of greater job instability. Furthermore, this position seems to be evolving toward the management of influencers.
\end{abstract}

\section{Financiación}

Esta investigación se ha implementado dentro del Proyecto Innova Docencia de la Universidad Complutense de Madrid (UCM-18-290) titulado Mapa visual de orientación profesional para el Grado de Publicidad y RRPP. 


\section{Keywords}

Professional profiles; Community managers; Community management; Social networks; Social media; Digital communication; Professional competences; Job offers.

\section{Introducción}

A pesar de que el interés por la especialización del community manager se ha promovido más en la bibliografía profesional que en la científica debido a una rápida trasformación necesaria para su adaptación a los cambios tecnológicos incesantes (Silva-Robles, 2016), en los últimos años se ha iniciado en España una línea investigadora que versa sobre el análisis de los perfiles y competencias profesionales requeridos en las áreas relacionadas con la Comunicación, junto a la necesidad de integrar académicamente esta especialización en los planes de estudio (Alcántara-López, 2011), que no alcanza aún ni al 5\% en las áreas de Comunicación de las universidades españolas (Navío-Navarro; González-Díez; Puebla-Martínez, 2018).

El camino se inicia en esta década como consecuencia de la dificultad para planificar la formación reglada y continúa en un contexto de digitalización constante (Vivar-Zurita, 2011) y la necesidad de implementar las competencias profesionales más allá de las habilidades y el conocimiento práctico (Perlado-Lamo-De-Espinosa, 2013). Desde los primeros años ya destaca la figura del community manager dentro de los perfiles profesionales vinculados a la comunicación 2.0 (Sánchez-Gonzales; Méndez-Muros, 2013).

Las ofertas de empleo relacionadas con las redes sociales concentran cada vez más roles y responsabilidades, aunque con un salario cada vez menor (Duffy; Schwartz, 2018). Entre las funciones del community manager se encuentra la gestión de comunidades a partir de una combinación de conocimiento, influencia y credibilidad, siendo esencial publicar contenido efectivo y establecer y hacer crecer las relaciones entre la organización y sus públicos (Ozan, 2001), considerando la importancia de la planificación de la reputación online (Castelló-Martínez, 2010).

Entre los reclutadores no hay consenso al definir cuáles son las funciones asociadas al community manager (Silva-Robles, 2017) y se encuentra en auge la petición de conocimientos y habilidades como los programas de diseño gráfico, gestores de contenido web (content management), posicionamiento SEO/SEM, publicidad digital, edición de vídeo, programas de gestión de redes sociales, etc. (Álvarez-Flores; Núñez-Gómez; Olivares-Santamarina, 2018).

A pesar del crecimiento de los chatbots para automatizar conversaciones con los usuarios (Serrano-Cobos, 2016), entre las cuatro competencias más utilizadas por los profesionales del marketing, la publicidad y la comunicación (IAB, 2018a) se encuentran la community management $(32,7 \%)$ y la gestión de contenidos $(28,4 \%)$. Precisamente por la capacidad de escuchar, dialogar y conectar personas que se requiere a este perfil profesional, la formación previa solicitada ha sido tan dispersa como Comunicación, Periodismo, Marketing, Humanidades, Administración de Empresas o algún ciclo formativo relacionado (Leiva-Aguilera, 2010).

El desempeño en social media es aún reciente, como demuestra el hecho de que en 2012 ocho de las 35 empresas españolas cotizadas en el IBEX 35 aún no utilizaran redes sociales (Almansa-Martínez; Godoy-Martín, 2012). Aunque en sus inicios el perfil mayoritario de community manager era el de menores de 40 años con licenciaturas de Marketing (Silva-Robles, 2012), rápidamente se inició una especialización ligada a la Comunicación, con una reducción progresiva de los tramos de edad requeridos.

En las pymes vascas y navarras se constató en el quinquenio 2013-2017 que la función de community manager había evolucionado desde un perfil de hombre mayor de 35 años con licenciatura afín a la Comunicación al de mujer menor de 35 años con formación específica en la materia, identificando con la percepción de los profesionales del sector un exceso de intrusismo profesional sin la cualificación adecuada (Elorriaga-Illera; Usin-Enales; García-Azpuru, 2018).

El community manager es un puesto de trabajo que a menudo se subcontrata a agencias de comunicación especializadas (Aced-Toledano, 2013), en las que la autonomía creativa acaba siendo limitada por la cultura corporativa de las empresas clientes (Kerr; Kelleher, 2015). El principal servicio que las empresas esperan por parte de las agencias de comunicación digital es el de la creación de contenidos (Scopen, 2017) y el 62,5\% de los dircoms considera que las áreas más destacadas son la comunicación online y los medios sociales (Dircom, 2018).

El análisis del perfil de community manager específico en agencias de comunicación refleja, en la misma línea, una presencia mayoritaria de mujeres menores de 35 años, con licenciaturas en Periodismo (42,6\%), Publicidad y RRPP (27,7\%) o Marketing (22,3\%) y con formación y experiencia previa digital (Silva-Robles, 2016).

La crisis económica y de los medios convencionales ha incentivado un trabajo de bajo coste, también en lo online, a pesar de que los futuros perfiles profesionales exigirán un mayor dominio del entorno tecnológico (Álvarez-Monzoncillo; Suárez-Bilbao; De-Haro, 2016). En este contexto desfavorable, el community manager debe afrontar un desempeño individual del puesto de trabajo que se resiente por un uso excesivo de las redes sociales (Yu et al., 2018), que es su instrumento esencial de trabajo, puesto que las profesiones asociadas a las nuevas tecnologías en general, y a las redes sociales en particular, están sujetas a mayores niveles de tecnoestrés (Brooks; Califf, 2017). 
Los continuos cambios a los que están expuestos los perfiles profesionales digitales hacen necesario un análisis con una perspectiva científica a través de la última década, que posibilita también comparar el auge de la figura del community manager en el inicio y el final de la crisis económica y de los medios sufrida, además de poder comprobar si el community manager ha ido absorbiendo otros perfiles digitales tradicionalmente separados, como el de creador de contenidos digitales (Núñez; García; Abuín, 2012).

\section{Objetivos y método}

Esta investigación tiene como objetivo general analizar la evolución del perfil profesional del community manager durante la década 2009-2018 a través de las ofertas de empleo.

La hipótesis de partida es que la gestión de las redes sociales en los perfiles profesionales digitales ha evolucionado hacia un perfil profesional específico de community manager, con funciones diferenciadas respecto a las de otros perfiles digitales.

Los objetivos específicos son:

- Analizar la evolución conceptual en su denominación.

- Determinar los cambios experimentados en las competencias y habilidades requeridas.

- Definir si evoluciona hacia su profesionalización o hacia una mayor precariedad.

- Establecer si hay diferencias significativas entre los perfiles.

Para ello se realiza un análisis de contenido de las ofertas de empleo vinculadas a la gestión de redes sociales -incluso cuando no aparezca en el titular de la oferta- que hayan sido publicadas entre enero de 2009 y diciembre de 2018 -año en curso con datos cerrados a 5 de diciembre- en domestika.org, señalado por la bibliografía científica como el principal portal especializado en las áreas que integran la Comunicación (Álvarez-Flores; Núñez-Gómez; Olivares-Santamarina, 2018). De este modo es posible analizar la evolución del perfil profesional del community manager a pesar de la evolución conceptual que haya experimentado y los cambios que se hayan producido en su denominación durante la última década.

Para comparar este perfil con otros perfiles de la comunicación digital, se han elaborado dos muestras:

- la primera, con 642 ofertas de empleo seleccionadas de forma aleatoria, es una muestra representativa y estratificada en años - para un nivel de confianza del $99 \%$ y bajo el supuesto de que $p=q=50 \%-$ de las 17.970 ofertas publicadas en domestika.org entre 2009 y 2018;

- la segunda está compuesta por las 731 ofertas de empleo que incorporan tareas de community manager publicadas en domestika.org durante el período 2009-2018. De éstas (tabla 1), el 74,97\% corresponden a un perfil exclusivo de community manager, frente a aquellas ofertas donde la gestión de una comunidad a través de las redes sociales no es la tarea central del perfil. Se considera exclusivo al perfil profesional que tiene como principal tarea la gestión de comunidades digitales a través de redes sociales.

Tabla 1. Ofertas de empleo del perfil community manager (CM) publicadas en domestika.org

\begin{tabular}{|c|c|c|c|c|c|c|}
\hline Año & $\begin{array}{l}\text { Universo } \\
\mathrm{n}=17.970\end{array}$ & $\begin{array}{c}\text { Muestra represen- } \\
\text { tativa estratifica- } \\
\text { da } n=642\end{array}$ & $\begin{array}{c}\text { Muestra de CM } \\
\qquad n=731\end{array}$ & $\begin{array}{c}\% \text { de ofertas de } \\
\text { CM respecto al } \\
\text { Universo }\end{array}$ & $\begin{array}{l}\text { Muestra de } C M \\
\text { exclusivo } n=548\end{array}$ & $\begin{array}{l}\text { \% de ofertas de } \\
\text { CM exclusivo res- } \\
\text { pecto al Universo }\end{array}$ \\
\hline 2009 & 690 & 25 & 10 & 1,45 & 4 & 0,58 \\
\hline 2010 & 1.320 & 47 & 28 & 2,12 & 20 & 1,52 \\
\hline 2011 & 2.280 & 81 & 69 & 3,03 & 51 & 2,24 \\
\hline 2012 & 1.380 & 49 & 55 & 3,99 & 35 & 2,54 \\
\hline 2013 & 1.140 & 41 & 28 & 2,46 & 20 & 1,75 \\
\hline 2014 & 1.410 & 50 & 50 & 3,55 & 40 & 2,84 \\
\hline 2015 & 1.950 & 70 & 77 & 3,95 & 51 & 2,62 \\
\hline 2016 & 2.310 & 83 & 111 & 4,81 & 84 & 3,64 \\
\hline 2017 & 2.280 & 81 & 138 & 6,05 & 109 & 4,78 \\
\hline 2018 & 3.210 & 115 & 165 & 5,14 & 134 & 4,17 \\
\hline Total & 17.970 & 642 & 731 & 36,5 & 548 & 26,6 \\
\hline
\end{tabular}

El análisis de cada oferta de empleo se realiza mediante una búsqueda selectiva de frases y palabras clave combinadas y un posterior proceso de desambiguación del lenguaje natural, utilizando

"patrones de manejo sintáctico que reflejan conocimiento léxico y sintáctico, las reglas ponderadas que reflejan conocimiento sintáctico, y la red semántica que refleja conocimiento semántico de cercanía de sentido entre grupos sintácticos" (Galicia-Haro; Gelbukh; Bolshakov, 2001, p. 63). 
Este tipo de análisis de contenido masivo es el idóneo para evitar un sesgo epistemológico habitual en este tipo de investigaciones, en los que es frecuente una descontextualización de los elementos que son significativos (Schroder, 2014) debido al elevado tamaño de la muestra. Por ello el análisis de contenido complementado con el análisis estadístico facilita comprender el contexto y la complejidad de los resultados obtenidos (Della-Porta; Keating, 2013), evitando la propensión a extrapolar casos particulares como significativos, a pesar de que estadísticamente puedan representar la mediana o la media aritmética de la muestra.

Las variables de análisis han sido:

- título de la oferta de empleo

- fecha de publicación

- empresa

- ciudad

- país

- tipo de empleo (beca, tiempo parcial, media jornada, tiempo completo)

- idioma de la oferta

- descripción (requisitos, condiciones de la oferta)

- salario anual bruto.

Mediante la prueba de Chi-cuadrado se determina si las diferencias estadísticas entre los grupos community manager exclusivo y no exclusivo son significativas.

\section{Resultados y discusión}

\subsection{Evolución de los perfiles profesionales de gestión de redes sociales}

Las ofertas de empleo publicadas que recogen la gestión de comunidades en redes sociales se han incrementado durante la década 2009-2018 (tabla 1), pero esa evolución ha sido significativa para el perfil exclusivo del community manager (tabla 2 y figura 1 ).

El porcentaje de ofertas no específicas de community manager que agregan tareas de gestión de redes sociales se ha mantenido estable en los últimos años. Estas funciones han tenido múltiples denominaciones en las ofertas de empleo durante la década estudiada, circunstancia habitual en los perfiles profesionales digitales emergentes, que evolucionan hasta alcanzar su madurez, corroborando así las teorías sobre el cambio conceptual (Bello-Garcés, 2004).
La gestión de las redes sociales ha estado ligada en los últimos 10 años a otras tareas como la gestión de marca o la redacción de contenidos
Tabla 2. Ofertas de empleo del perfil community manager publicadas en domestika.org

\begin{tabular}{|c|c|c|}
\hline Año & $\begin{array}{c}\text { \% ofertas community } \\
\text { manager no exclusivo } \\
\mathbf{n = 1 8 3}\end{array}$ & $\begin{array}{c}\text { \% ofertas community } \\
\text { manager exclusivo } \\
\mathbf{n}=\mathbf{5 4 8}\end{array}$ \\
\hline 2009 & 0,87 & 0,58 \\
\hline 2010 & 0,61 & 1,52 \\
\hline 2011 & 0,79 & 2,24 \\
\hline 2012 & 1,45 & 2,54 \\
\hline 2013 & 0,70 & 1,75 \\
\hline 2014 & 0,71 & 2,84 \\
\hline 2015 & 1,33 & 2,62 \\
\hline 2016 & 1,17 & 3,64 \\
\hline 2017 & 1,27 & 4,78 \\
\hline 2018 & 0,97 & 4,17 \\
\hline Total & $\mathbf{9 , 8 7}$ & $\mathbf{2 6 , 6 8}$ \\
\hline
\end{tabular}

El concepto community manager y otras acepciones ligadas a la idea de comunidad-gestor de comunidades o dinamizador de comunidad, por ejemplo- son las denominaciones que más se han utilizado para referirse a este perfil (figura 2), con la excepción puntual del año 2016, en el que fue superada por social media, una denominación que agrupa perfiles con el acento en las redes sociales -social media manager, social media strategist, gestión de redes sociales o dinamizador de

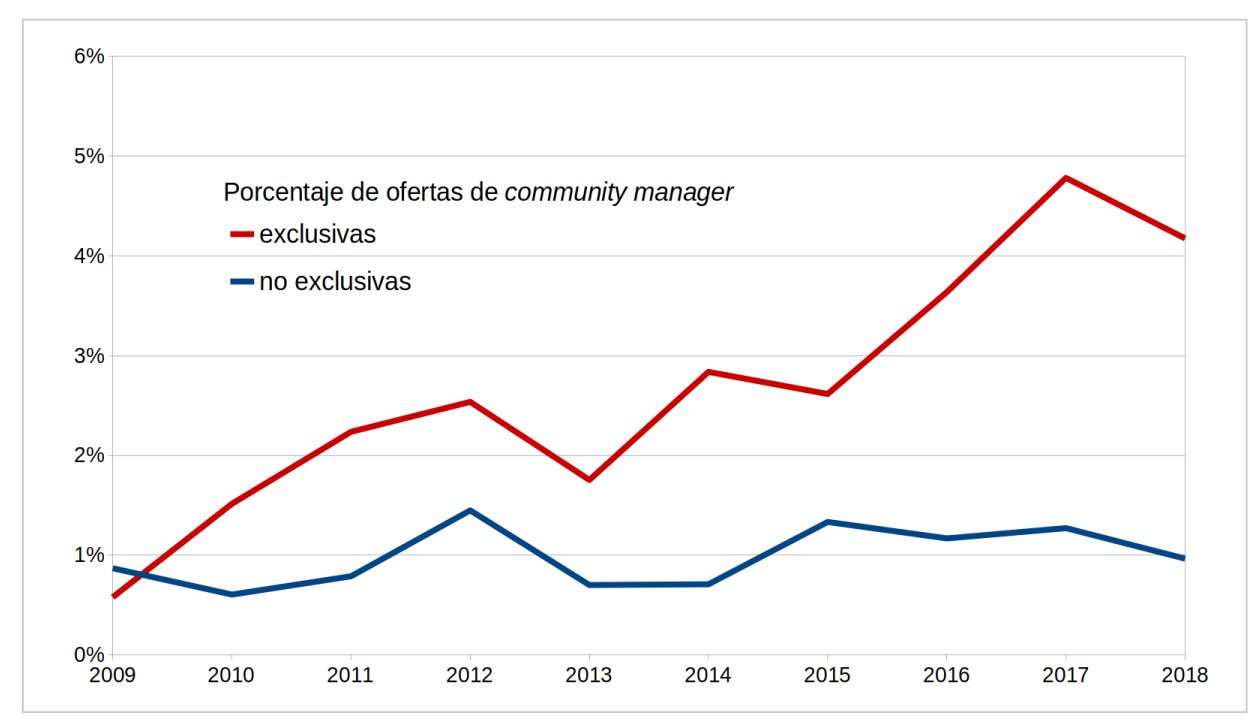

Figura 1. Evolución del perfil del community manager en domestika.org 
redes sociales-. A pesar de ello, en buena parte de los perfiles considerados se utiliza community manager y social media manager como sinónimos.

De hecho, y a pesar de este predominio de los perfiles que ponen el foco en la comunidad y en las redes sociales, en los últimos años se encuentran en auge los perfiles vinculados al marketing y la estrategia digital, revelando el análisis cualitativo que cada vez más se establecen como requisitos elaborar la estrategia, creatividad y gestión de campañas publicitarias - ads- en redes sociales. El uso de anglicismos es habitual en las ofertas de empleo de perfiles digitales y en escasas ocasiones se traducen estos términos.

En cuanto al incremento experimentado en los últimos cinco años por la creación y gestión de contenidos, gracias en parte al auge del branded content, los perfiles de content writer o content manager suponen una reformulación, dentro del marketing digital, del copywriter publicitario, que figura a continuación en los perfiles más demandados en las ofertas de empleo.

El análisis de las ofertas de empleo también revela que la gestión de las redes sociales ha estado ligada en los últimos 10 años a otras tareas del ecosistema digital, como la gestión de marca o la redacción de contenidos para campañas de marketing. A pesar de ello, en los primeros años examinados, esa vinculación aparecía con tareas menos relacionadas con la comunicación y el marketing y la gestión de las redes sociales correspondía al programador o diseñador de sitios web.

En el inicio de la década, las competencias profesionales del community manager estaban más orientadas hacia la tecnología que hacia los conocimientos, habilidades y competencias de un especialista en comunicación. La tendencia a asociar las tareas de gestión de las redes sociales y la ejecución de la estrategia de marketing y comunicación en el entorno digital parece apuntar a la importancia creciente que han tenido estos medios sociales en la comunicación de las organizaciones.

También se aprecia una especialización sectorial, a partir de la cual se demanda la contratación de community managers con especialización temática en el sector de los videojuegos, salud, belleza y moda o en el de los automóviles. En último lugar se produce una especialización por red social que aproxima cada vez más la figura del community manager a la de influencer al demandar, por ejemplo, expertos en YouTube o en Instagram.

\subsection{Consolidación de la precariedad laboral}

Un $26,54 \%$ de las ofertas de empleo de gestor de redes sociales revisadas están ligadas a contratos para los jóvenes que se incorporan al mundo laboral, frente al 20,25\% del total de las ofertas en domestika.org, y sólo un

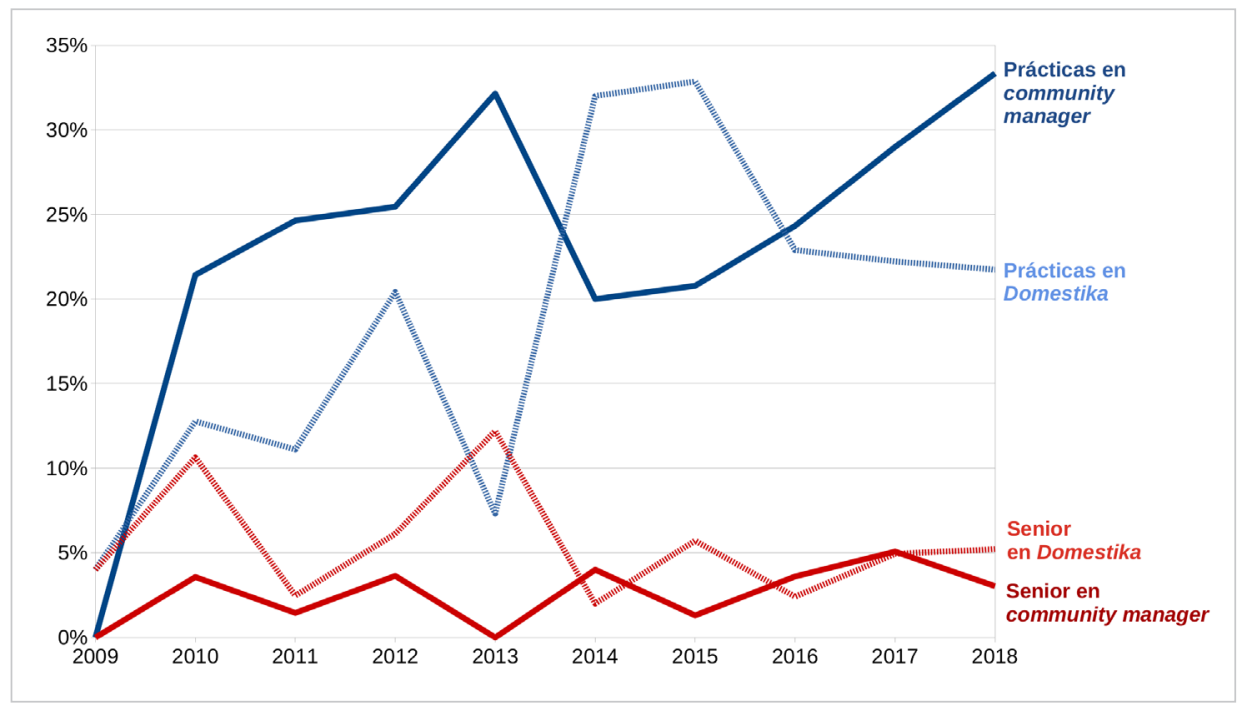

Figura 3. Evolución de las ofertas de empleo de domestika.org y del gestor en redes sociales con perfil en prácticas y senior 
$3,15 \%$ se dirige a un profesional senior, frente al 5,14\% del total de las ofertas publicadas. La contratación de community managers mediante convenios de prácticas con las universidades y otros centros de formación muestra una tendencia al alza (figura 3 ), sobre todo en los primeros años de crisis y en los últimos años de recuperación económica, dato preocupante en cuanto a la profesionalización y adecuada remuneración de este perfil profesional.

\subsection{Competencias profesionales}

A pesar de que las figuras de contratación del community manager apuntan hacia la precariedad, cada año aumentan los requisitos de las ofertas de empleo en cuanto a las competencias profesionales que debe reunir, muchas de ellas compartidas con otros perfiles digitales.

El dominio del idioma inglés es uno de los requisitos básicos, con un $55,13 \%$ de las ofertas de empleo de gestor de redes sociales que lo exige, frente al 32,09\% del total de las ofertas de domestika.org. Este requisito se ha duplicado en la última década (figura 4), superando el $60 \%$ en las ofertas de empleo de community manager.

También el número de ofertas escritas en inglés es algo superior en el perfil del gestor de redes sociales que en el resto de los perfiles de domestika.org, dato que se encuentra en consonancia con el uso habitual de términos anglosajones en las funciones de los perfiles digitales.

En cuanto a las redes sociales sobre las que se exigen conocimientos, destacan Facebook, en un 34,20\% de las ofertas de gestor de redes sociales, y Twitter, en un 28,45\% (tabla 3). Instagram, LinkedIn, YouTube y Pinterest forman un segundo grupo de redes sociales con menor presencia, mientras que el impacto de Snapchat y WhatsApp es muy reducido.

Tabla 3. Ofertas de empleo del perfil community manager publicadas en domestika.org

\begin{tabular}{|c|c|c|c|c|c|c|c|c|}
\hline Red social & Facebook & Twitter & Instagram & Linkedln & YouTube & Pinterest & Snapchat & WhatsApp \\
\hline$N^{\circ}$ de ofertas en las que se pide & 250 & 208 & 138 & 92 & 88 & 69 & 10 & 1 \\
\hline$\%$ respecto al total & 34,20 & 28,45 & 18,88 & 12,59 & 12,04 & 9,44 & 1,37 & 0,14 \\
\hline
\end{tabular}

Si atendemos a la evolución de estas redes sociales en las ofertas de empleo (figura 5), Facebook y Twitter mantienen una presencia más o menos estable, mientras que Instagram muestra un notable crecimiento en los últimos años, lo cual corrobora los datos que la sitúan como la red social que más crece y la que más incrementa su frecuencia de visita, habiendo adelantado ya a Twitter en penetración de usuarios $(I A B, 2018 b)$.
La gestión de redes sociales ha dejado de corresponder a programadores y diseñadores web para convertirse en un perfil profesional de Comunicación

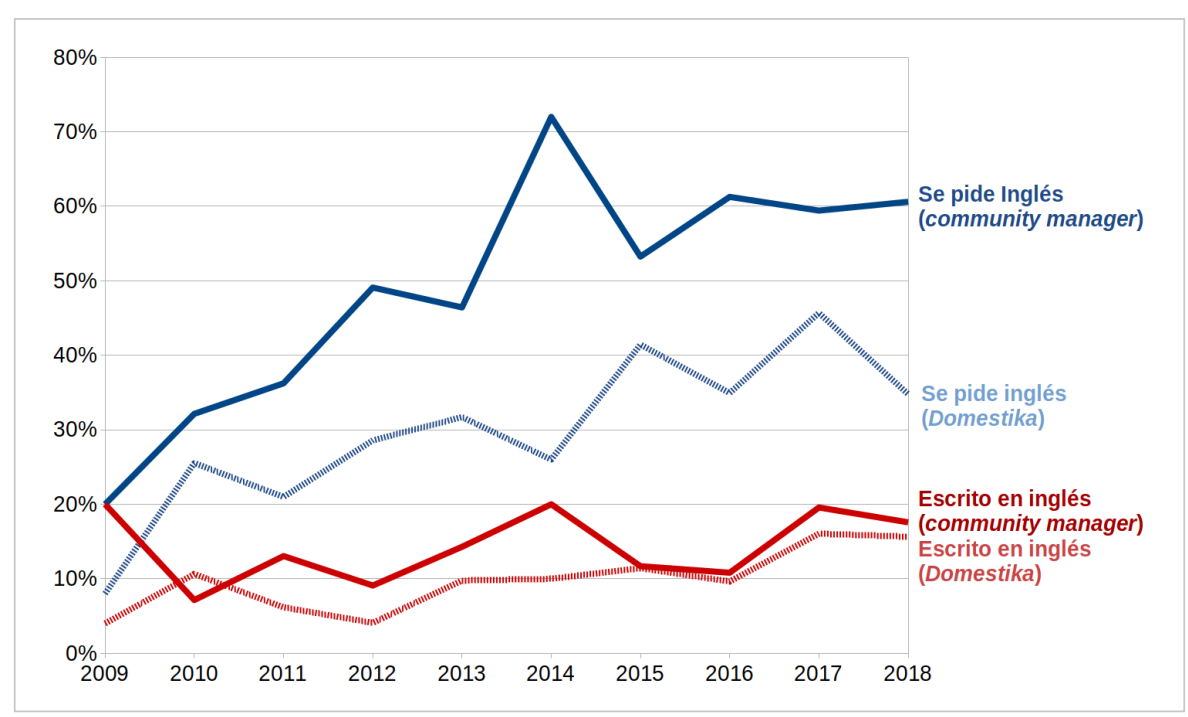

Figura 4. Evolución del requisito del idioma inglés en las ofertas de empleo publicadas en domestika.org 
En cuanto a las singularidades específicas del gestor de redes sociales, encontramos la capacidad de redacción y generación de contenidos como la más solicitada $(44,32 \%$ de las ofertas de empleo), seguida de cerca de la interacción y atención al cliente $(40,77 \%)$ y, por último, la gestión y dinamización de la comunidad (17,65\%). Esta última tarea, que fue clave en los primeros perfiles de community manager, ha ido quedando relegada en estos últimos años (figura 6).

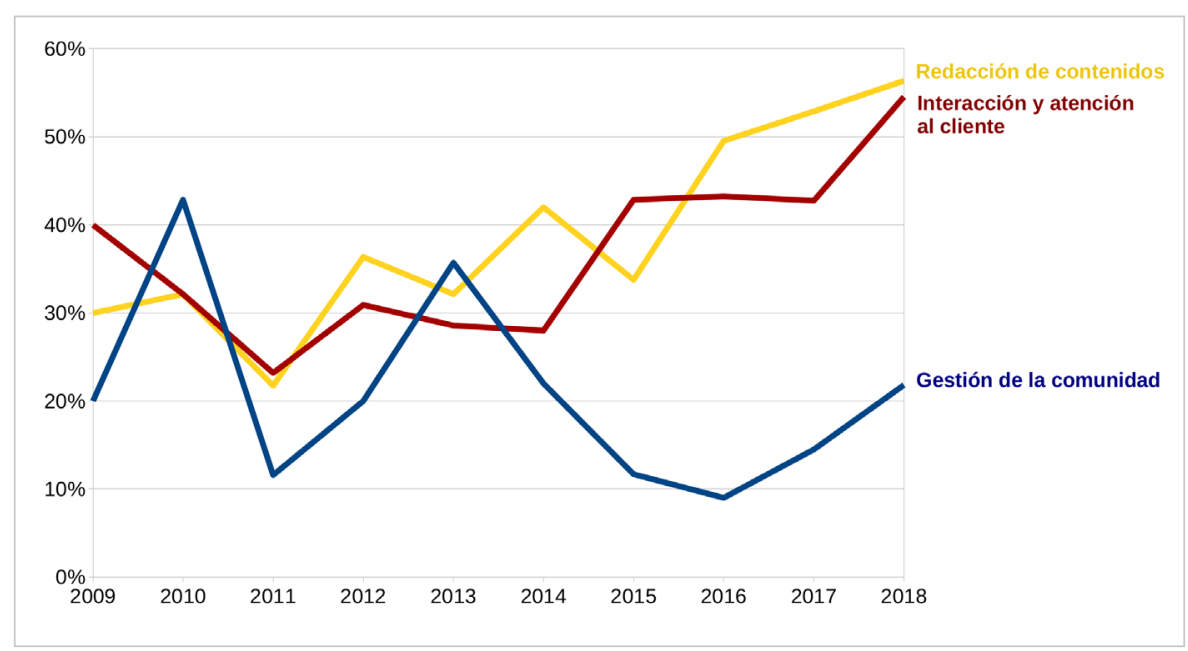

Figura 6. Evolución de las principales funciones específicas del gestor de redes sociales en domestika.org

Al analizar estas funciones en el perfil del community manager exclusivo, observamos que la redacción de contenidos y la gestión de la comunidad son requisitos significativamente más frecuentes $(p<0,05)$ en este perfil que en el del gestor de redes sociales no exclusivo. La interacción y atención al cliente (tabla 4) es una función compartida en ambos perfiles, ya que no muestra diferencias significativas.

Tabla 4. Estadístico Chi-cuadrado $\chi^{2}$ de las principales funciones específicas del gestor de contenidos en el community manager exclusivo y en el no exclusivo

\begin{tabular}{|c|c|c|c|c|}
\hline $\begin{array}{c}\text { Función demandada } \\
\text { en el perfil }\end{array}$ & $\begin{array}{l}\text { Frecuencias en el perfil de commu- } \\
\text { nity manager exclusivo } \\
\qquad(n=548)\end{array}$ & $\begin{array}{l}\text { Frecuencias en el perfil de com- } \\
\text { munity manager no exclusivo } \\
\qquad(n=183)\end{array}$ & $\chi^{2}$ & $\begin{array}{l}\text { P-value } \\
(p<0,05) \\
(p>0,05)\end{array}$ \\
\hline Redacción de contenidos & $258(242,89)$ & $66(81,11)$ & 6,7446 & 0,009403 \\
\hline Interacción y atención al cliente & $223(223,4)$ & $75(74,6)$ & 0,0048 & 0,944859 \\
\hline Gestión y dinamización de la comunidad & $112(96,71)$ & $17(32,29)$ & 11,7323 & 0,000614 \\
\hline
\end{tabular}

La evolución de las dos funciones significativas en el perfil del community manager exclusivo es diferente, sobre todo en los primeros años, con respecto a la del no exclusivo (figura 7). Así, la capacidad para redactar contenidos se convierte en estos últimos años en la función diferenciadora del community manager exclusivo.

Con todo, la evolución del requisito de interacción y atención al cliente en el perfil del community manager exclusivo es relevante, porque podría explicar la tendencia en

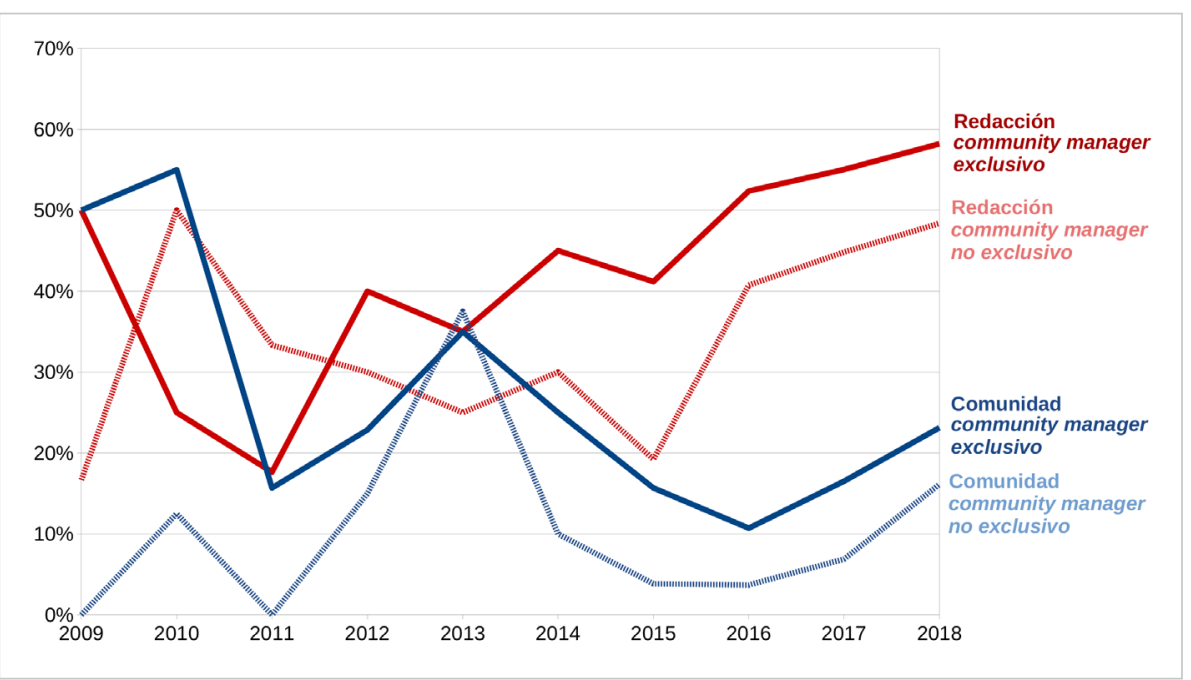

Figura 7. Evolución de las dos funciones significativas del community manager exclusivo todos los perfiles de gestión de redes sociales hacia el marketing digital (figura 8).

En efecto, al analizar otras funciones del perfil vinculadas al marketing digital, observamos un notable crecimiento (figura 9) en el posicionamiento -SEO, SEM y de marca-, la gestión de influencers y, sobre todo, las estrategias de marketing y la elaboración de métricas.
La especialización del community manager le acerca cada vez más a la gestión de influencers 
Tabla 5. Estadístico Chi-cuadrado $\chi^{2}$ de las principales funciones específicas del gestor de contenidos en el community manager exclusivo y en el no exclusivo

\begin{tabular}{|l|c|c|c|c|}
\hline \multicolumn{1}{|c|}{$\begin{array}{c}\text { Función demandada } \\
\text { en el perfil }\end{array}$} & $\begin{array}{c}\text { Frecuencias en el perfil de com- } \\
\text { munity manager exclusivo } \\
\mathbf{( n = 5 4 8 )}\end{array}$ & $\begin{array}{c}\text { Frecuencias en el perfil de } \\
\text { community manager no exclu- } \\
\text { sivo (n=183) }\end{array}$ & $\begin{array}{c}\boldsymbol{P} \text {-value } \\
\mathbf{( p )}\end{array}$ \\
\hline Gestión de influencers & $55(46,48)$ & $7(15,52)$ & 6,8187 & 0,009021 \\
\hline Posicionamiento (SEO, SEM, y de marca) & $126(138,69)$ & $59(46,31)$ & 6,2066 & 0,012727 \\
\hline Métricas y estadísticas & $259(251,14)$ & $76(83,86)$ & 1,8161 & 0,177783 \\
\hline Estrategias de marketing & $359(365,83)$ & $129(122,17)$ & 1,5337 & 0,215563 \\
\hline
\end{tabular}

A pesar de ello, tanto las estrategias de marketing como la generación de métricas presentan en ambos grupos (tabla 5) frecuencias que no son estadísticamente significativas, es decir, que son frecuentes por igual en ambos tipos de perfil. Sin embargo, la gestión de influencers tiene una presencia importante en el perfil del community manager exclusivo $(p=0,009021)$ $y$ el posicionamiento SEO, SEM y de marca, lo tiene en el perfil del community manager no exclusivo ( $p=0,012727)$.

Por tanto, aunque el marketing ha ganado importancia en el perfil actual del community manager, todo apunta a que hay funciones que encajan mejor que otras. Es el caso de la gestión de los influencers, que ha pasado a ser una tarea asignada al community manager exclusivo (véase figura 10).

\section{Conclusiones}

A pesar de que la especialización del community manager no está presente ni en el $5 \%$ de los planes de estudio de los grados en Comunicación, el 75\% de las ofertas que contienen tareas de community manager corresponde a un perfil exclusivo y diferenciado de otros digitales. Estas ofertas con perfil específico se han incrementado durante los últimos cinco años.

El community manager se ha ido desprendiendo con el paso de los años de algunas tareas asociadas a su perfil, como el diseño de webs. Sin embargo, ahora asume nuevas funciones asociadas al marketing digital, posicionamiento, analítica, rela-

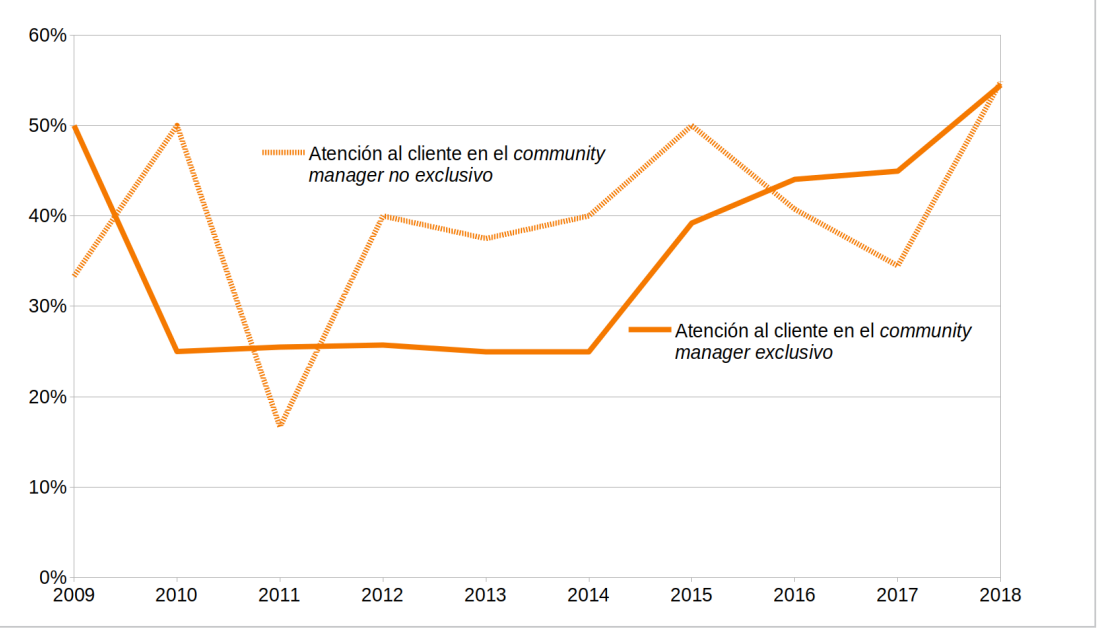

Figura 8. Evolución de las dos funciones principales significativas del community manager exclusivo

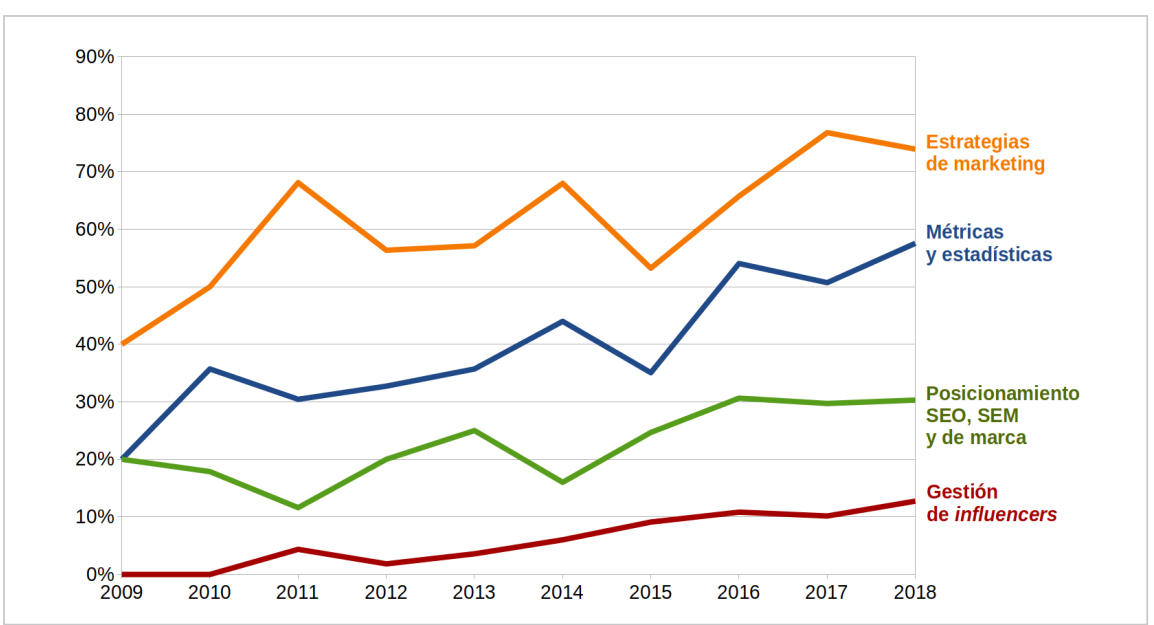

Figura 9. Evolución de funciones vinculadas al marketing en el perfil del community manager

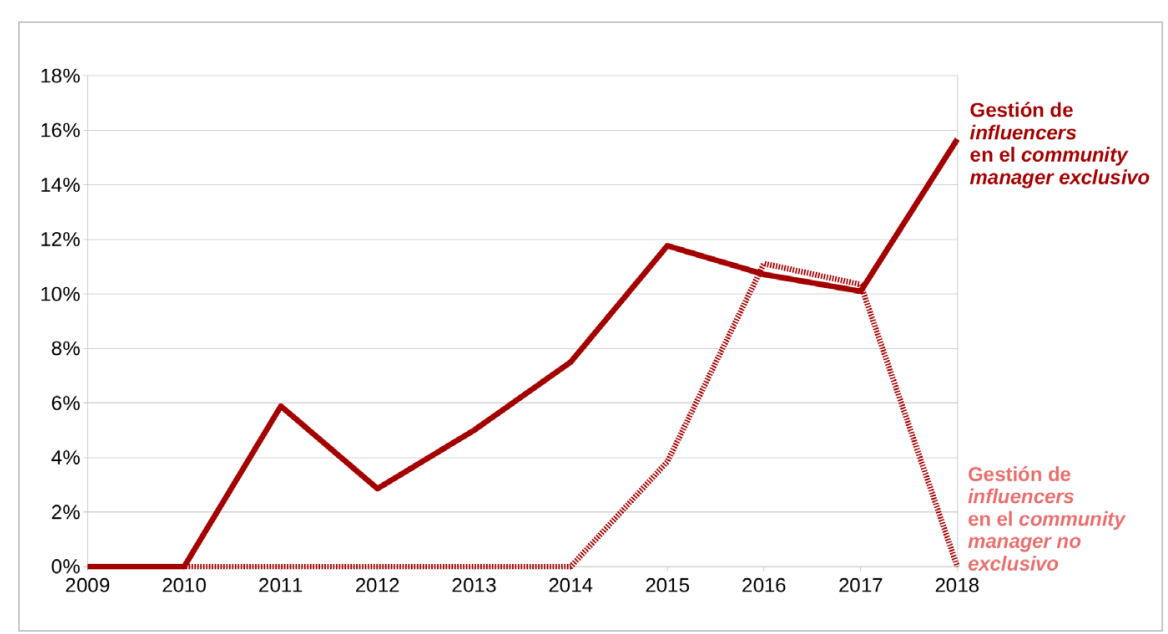

Figura 10. Evolución de la gestión de influencers en el perfil del community manager 
ciones públicas o gestión de marca, por lo que la hipótesis de partida queda sólo parcialmente validada.

De hecho, la capacidad de gestión de la comunidad, tarea esencial en el perfil durante los primeros años, ha perdido importancia frente a la capacidad para generar contenidos y a otras tareas más próximas al marketing digital, como la atención al cliente y las relaciones con los stakeholders. El dominio del inglés es requisito en el $60 \%$ de las ofertas de empleo, casi el doble de lo exigido por el total de las ofertas.

El uso actual en las ofertas de empleo de denominaciones como community manager, social media manager o digital manager como si fueran sinónimos, además de generar cierta confusión, denota la búsqueda de perfiles profesionales que dominen completamente el área digital. Se confirma así la exigencia de un mayor número de competencias y habilidades asociadas a este perfil, aunque en un contexto de mayor precariedad laboral. Sólo el $3 \%$ de las ofertas de community manager buscan a un profesional senior.

Sin embargo, algunas tareas, como la comunicación con los influencers, ligada en parte a las relaciones públicas, son en las ofertas de empleo parte integrante del perfil del community manager exclusivo. El crecimiento que Instagram ha experimentado durante los últimos años como requisito en las ofertas de empleo corrobora esta situación.

Entre las futuras líneas de investigación es necesario profundizar en la tendencia del community manager hacia la gestión del influencer, comparando las ofertas de empleo españolas con las de otros países más avanzados en cuanto a perfiles profesionales digitales.

\section{Referencias}

Aced-Toledano, Cristina (2013). Relaciones públicas 2.0. Cómo gestionar la comunicación corporativa en el entorno digital. Barcelona: UOC. ISBN: 9788490292495

Alcántara-López, Rocío (2011). "La comunicación como herramienta clave del community manager. Justificación de su presencia en las facultades de Comunicación". Vivat academia, v. 14, n. 117E, pp. 1405-1416.

https://doi.org/10.15178/va.2011.117E.1405-1416

Almansa-Martínez, Ana; Godoy-Martín, Francisco-Javier (2012). "El community manager en las principales empresas de España: una aproximación a su formación y su situación laboral”. Estudios sobre el mensaje periodístico, v. 18, pp. 57-65. https://doi.org/10.5209/rev_ESMP.2012.v18.40887

Álvarez-Flores, Erika-Patricia; Núñez-Gómez, Patricia; Olivares-Santamarina, José P. (2018). “Perfiles profesionales y salidas laborales para graduados en Publicidad y Relaciones públicas: de la especialización a la hibridación". El profesional de la información, v. 27, n. 1, pp. 136-147.

https://doi.org/10.3145/epi.2018.ene.13

Álvarez-Monzoncillo, José-María; Suárez-Bilbao, Fernando; De-Haro, Guillermo (2016). “Challenges and considerations of the new labor market in the media industry". El profesional de la información, v. 25, n. 2, pp. 262-271. https://doi.org/10.3145/epi.2016.mar.13

Bello-Garcés, Silvia (2004). “Ideas previas y cambio conceptual”. Educación química, v. 15, n. 3, pp. $210-217$. https://doi.org/10.22201/fq.18708404e.2004.3.66178

Brooks, Stoney; Califf, Christopher (2017). "Social media-induced technostress: Its impact on the job performance of IT professionals and the moderating role of job characteristics". Computer networks, v. 114, pp. 143-153. https://doi.org/10.1016/j.comnet.2016.08.020

Castelló-Martínez, Araceli (2010). "Una nueva figura profesional: el community manager". Pangea: Revista de la Red Académica Iberoamericana de Comunicación, v. 1, n. 1, pp. 74-97.

https://bit.ly/29Yz5kc

Della-Porta, Donatella; Keating, Michael (2013). Enfoques y metodologías de las ciencias sociales. Una perspectiva pluralista. Madrid: Akal. ISBN: 9788446030621

Dircom (2018). Estado de la Comunicación en España 2018. Asociación de Directivos de Comunicación. https://bit.ly/2PfKkXf

Duffy, Brooke-Erin; Schwartz, Becca (2018). “Digital 'women's work?': Job recruitment ads and the feminization of social media employment”. New media \& society, v. 20, n. 8, pp. 2972-2989.

https://doi.org/10.1177/1461444817738237

Elorriaga-Illera, Angeriñe; Usin-Enales, Sandra; García-Azpuru, Amaia (2018). “Evolución del community manager en las pymes del País Vasco y Navarra". El profesional de la información, v. 27, n. 3, pp. 651-658. 
https://doi.org/10.3145/epi.2018.may.18

Galicia-Haro, Sofía N.; Gelbukh, Alexander; Bolshakov, Igor A. (2001). “Una aproximación para resolución de ambigüedad estructural empleando tres mecanismos diferentes". Procesamiento del lenguaje natural, v. 27, pp. 55-64.

https://bit.ly/2B6kgbE

IAB (2018a). Estudio del mercado laboral en marketing digital. IAB Spain.

https://bit.ly/2KRWcQT

IAB (2018b). Estudio anual de redes sociales 2018. IAB Spain.

https://bit.ly/2J95UhE

Kerr, Aphra; Kelleher, John D. (2015). "The recruitment of passion and community in the service of capital: Community managers in the digital games industry". Critical studies in media communication, v. 32, n. 3, pp. 177-192.

https://doi.org/10.1080/15295036.2015.1045005

Leiva-Aguilera, Javier (2010). “Comunicación en la empresa y apertura del perfil profesional de los documentalistas". El profesional de la información, v. 19, n. 2, pp. 117-121.

https://doi.org/10.3145/epi.2010.mar.01

Navío-Navarro, Mariché; González-Díez, Laura; Puebla-Martínez, Belén (2018). "La formación para la gestión de las redes sociales en los grados de comunicación en España y su adecuación a las competencias demandadas por las organizaciones". Doxa comunicación, n. 26, pp. 127-143.

https://doi.org/10.31921/doxacom.n26a6

Núñez, Patricia; García, Alberto; Abuín, Natalia (2012). “Profesionales digitales en publicidad y comunicación. Una aproximación a las necesidades del mercado laboral”. Cuadernos de información y comunicación, n. 18, pp. 177-187. https://doi.org/10.5209/rev_CIYC.2013.v18.41723

Ozan, Ayse-Yuksel (2001). "The community manager and the importance of community knowledge in e-marketing". Journal of the Institution of British Telecommunications Engineers, v. 2, n. 2, pp. 79-82.

Perlado-Lamo-de-Espinosa, Marta (2013). "Nuevas oportunidades en la comunicación digital: nuevos perfiles y competencia". Prospectivas y tendencias para la comunicación en el sigo XXI, v. 17, n. 6, pp. 429-440. ISBN: 9788415949176

Sánchez-Gonzales, Hada; Méndez-Muros, Sandra (2013). “¿Perfiles profesionales 2.0? Una aproximación a la correlación entre la demanda laboral y la formación universitaria”. Estudios sobre el mensaje periodístico, n. 19, pp. 981-993. https://doi.org/10.5209/rev_ESMP.2013.v19.42183

Schroder, Kim-Christian (2014). "Realidades discursivas". En: Jensen, Klaus-Bruhn. La comunicación y los medios. Metodologías de investigación cualitativa y cuantitativa. México D.F.: Fondo de Cultura Económica, pp. 152-18. ISBN: 978 6071626561

Scopen (2017). PR Scope España 2017.

https://bit.ly/2xFF1L7

Serrano-Cobos, Jorge (2016). "Tendencias tecnológicas en internet: hacia un cambio de paradigma". El profesional de la información, v. 25, n. 6, pp. 843-850.

https://doi.org/10.3145/epi.2016.nov.01

Silva-Robles, Carmen (2012). “Community managers: la dirección de RR.PP. en la Red”. Revista internacional de relaciones públicas, v. 2, n. 3, pp. 193-216.

https://doi.org/10.5783/RIRP-3-2012-10-193-216

Silva-Robles, Carmen (2016). "Perfil del community manager en las agencias de publicidad y relaciones públicas de España". El profesional de la información, v. 25, n. 2, pp. 237-245.

https://doi.org/10.3145/epi.2016.mar.10

Silva-Robles, Carmen (2017). "The community manager: Responsibilities assigned by companies”. En: Campos-Freire, Francisco; Rúas-Araujo, Xosé; Martínez-Fernández, Valentín-Alejandro; López-García, Xosé (2013). Media and metamedia management. Cham: Springer, pp. 271-277. ISBN: 9783319460666

https://doi.org/10.1007/978-3-319-46068-0_35

Vivar-Zurita, Hipólito (2011). “Nuevos perfiles profesionales para una comunicación digital”. Telos. Cuadernos de comunicación e innovación, n. 87, pp. 58-62.

https://telos.fundaciontelefonica.com/archivo/numero087/nuevos-perfiles-profesionales-para-una-comunicacion-digital

Yu, Lingling; Cao, Xiongfei; Liu, Zhinying; Wang, Junkai (2018). "Excessive social media use at work". Information technology \& people, v. 31, n. 6, pp. 1091-1112.

https://doi.org/10.1108/ITP-10-2016-0237 